\title{
Real-World Determinants of Adjunctive Antipsychotic Prescribing for Patients with Major Depressive Disorder and Inadequate Response to Antidepressants: A Case Review Study
}

Roger S. McIntyre · Emmanuelle Weiller

To view enhanced content go to www.advancesintherapy.com

Received: March 12, 2015 / Published online: May 13, 2015

(C) The Author(s) 2015. This article is published with open access at Springerlink.com

\begin{abstract}
Introduction: Patients with major depressive disorder (MDD) often fail to respto first-line antidepressant treatments (ADTs); subsequent strategies include dosage increase, switch to a different ADT, or addition of another ADT or other drug. The objective of this prospective, case review study was to identify factors that influence the decision to prescribe adjunctive antipsychotics for patients with MDD and inadequate response to ADT.
\end{abstract}

Methods: Psychiatrists or primary care physicians $(n=411)$ based in the USA and Europe each completed an online survey for ten consecutive adults with MDD and inadequate response to ADTs, and for whom a treatment change was considered. A $t$ test was

Electronic supplementary material The online version of this article (doi:10.1007/s12325-015-0207-3) contains supplementary material, which is available to authorized users.

R. S. McIntyre ( $\square)$

University of Toronto, Toronto, ON, Canada

e-mail: roger.mcintyre@uhn.ca

E. Weiller

H. Lundbeck A/S, Valby, Denmark used to compare survey responses between groups of patients.

Results: The survey was completed for 4018 patients; an adjunctive antipsychotic was considered for $961 / 4018$ patients $(23.9 \%)$ and actually prescribed for 514/4018 (12.8\%). Compared with patients not considered for an adjunctive antipsychotic, those who were considered for this treatment had more previous major depressive episodes (MDEs), longer duration of the current MDE, more severe illness both at ADT initiation and current consultation, and more treatment changes. Patients who were prescribed adjunctive antipsychotics had at baseline more functional impairment and absences from work than those considered for but not prescribed this treatment. Key symptoms that prompted physicians to consider antipsychotics were psychotic symptoms, psychomotor agitation, hostility, irritability, impulsivity, and anger bursts. Anxious mood and irritability were mentioned significantly more often by physicians who actually prescribed adjunctive antipsychotics. Obstacles to prescribing included a tendency to wait to see if symptoms improved and concern over side effects. 
Conclusion: This real-world study suggested that the decision to prescribe an adjunctive antipsychotic for patients with MDD and inadequate response to ADT is influenced by a broad spectrum of factors, predominantly related to severity of illness, functional impairment, and symptom profile.

Funding: Otsuka Pharmaceutical Development \& Commercialization, Inc. (Princeton, USA) and H. Lundbeck A/S (Valby, Denmark).

Keywords: Adjunctive; Antidepressants; Antipsychotics; Augmentation; Major depressive disorder; Psychiatry; Real world; Treatment decision

\section{INTRODUCTION}

Despite the availability of a range of antidepressant treatments (ADTs), many patients with major depressive disorder (MDD) only have a partial response to first-line therapies [1, 2]. An inadequate response is more likely to occur in patients with a longer duration of illness, more severe symptoms and comorbid illnesses [3]. Since incomplete remission is associated with an increased risk of relapse, impaired work and social functioning, and an increased risk of suicide [3], it is important that patients with an inadequate response are both promptly identified and prescribed an effective course of treatment.

Identification of patients with inadequate response can be achieved via the use of standardized and validated measurement tools, so that modifications to treatment, including dosage increases, changing ADT, or the addition of an adjunctive treatment, can be promptly implemented. Augmentation of treatment with adjunctive therapies can be either another ADT of the same or different class (combination therapy), or a new therapeutic approach such as administration of a second-generation antipsychotic [1, 4, 5] which has been shown to improve the clinical response rate at 6 weeks in patients with MDD refractory to ADT by as much as 50\% [6]. However, second-generation antipsychotics are frequently associated with intolerable side effects $[7,8]$.

With increasing numbers of both ADTs and second-generation antipsychotics indicated for use in MDD, the absence of evidence-based guidelines and lack of high-quality data [9] complicates the choice of adjunctive drug for patients with an inadequate response to monotherapy. The choice of treatment for any individual patient may depend on factors such as symptomatology, disease and treatment history, and patient preference. A retrospective study on choice of adjunctive treatment reported that augmentation of ADT with a second-generation antipsychotic was more likely in patients with the most severe depression [10].

The objective of this case review study was to examine real-world data from patient records to identify the key factors that influence the treatment decision for patients with MDD and an inadequate response to ADT.

\section{METHODS}

\section{Study Design}

This chart review study was designed to be prospective and was conducted in the USA and Europe between February and April 2014. Physicians invited to participate were psychiatrists or primary care physicians (USA only) who had been qualified for between 3 and 40 years and were actively managing and treating patients with MDD. An appropriate caseload was considered to be at 
least 20 patients with MDD in a typical month. The physicians were recruited from a specialist market research panel and their qualifications were subsequently verified. Physicians were invited to take part by email and those who responded answered several screening questions to ensure that they met the inclusion criteria. Physicians were selected to provide a balance of hospital- versus officebased and public versus private psychiatrists with a representative spread within key geographic regions.

Physicians were asked to complete an online survey that was divided into three sections (see the electronic supplementary material). Section A was designed to collect information about the physician, and estimates or perceptions of their caseload and general clinical management of patients with MDD. Section Bi was prospectively completed for ten consecutive adults, who the physician considered to have a diagnosis of MDD, who were being treated with ADTs for a current major depressive episode (MDE), who had an inadequate response, and for whom the physician considered or made a treatment change at the current consultation. Since the study objective was to understand the real-world clinical practice, International Classification of Diseases (ICD) or Diagnostic and Statistical Manual of Mental Disorders (DSM) diagnostic criteria were not specified as part of the patient selection process. Towards the end of recruitment, the protocol was modified so that physicians could complete the survey for between five and ten patients. Section Bii was only completed for patients for whom treatment with an adjunctive antipsychotic was considered or prescribed. Sections Bi and Bii were completed using information from the consultation and patient record forms on the day of consultation, or as soon as possible afterwards.
Section Bi comprised questions on the patient's socio-demographic characteristics, disease and treatment history, duration and severity of current MDE (based on Clinical Global Impression-Severity [CGI-S]), comorbidities, current treatment, change to treatment regimen considered or initiated at current consultation, reasons for prescribing the new treatment, and barriers to the use of adjunctive antipsychotics in this patient. Physicians who indicated that they preferred to reserve antipsychotics for specific symptoms were asked to select from a checklist the symptoms that would trigger prescription of this class of drug. Physicians who stated they had tolerability or safety concerns that prevented them from adding an antipsychotic for a particular patient were asked to identify their concerns from a checklist.

Section Bii included questions on which factors had led to prescription or consideration of adjunctive antipsychotics for each patient. Questions regarding symptoms that led to adjunctive antipsychotic prescribing were presented randomly to half the physicians as a symptom checklist (coded) and half as an open text box (open) to evaluate any potential effect of prompted compared with non-elicited responses.

The analysis in this article is based on patient record forms, in the form of market research with physician and patient anonymity preserved entirely, and does not involve any new studies with human or animal subjects performed by any of the authors. Consequently, institutional review board approval and patient informed consent were not required.

\section{Statistical Analysis}

Data relating to patients have been summarized for: all patients (Bi); patients for whom an 
adjunctive antipsychotic was considered or actually prescribed (Bii: adjunctive antipsychotic group); and patients for whom an adjunctive antipsychotic was not considered or prescribed (Bi-Bii: non-adjunctive antipsychotic group).

Statistical analyses were: demographic and disease characteristics for the adjunctive antipsychotic-considered and adjunctive antipsychotic-prescribed groups compared with the non-adjunctive antipsychotic group; employment status, absenteeism, presenteeism and impact on social functioning parameters compared between the adjunctive antipsychotic-considered and adjunctive antipsychotic-prescribed groups; symptoms that triggered prescription of antipsychotics compared between physicians who did not and did actually prescribe this treatment to patients in the survey; comparison of symptoms captured using the coded and open formats (where open questions were coded up into categories so that these could be treated as quantitative data). All statistical comparisons were made using a $t$ test at a significance level of 0.05 .

\section{RESULTS}

\section{Physicians}

The survey was completed by 411 physicians located in the USA $(129,31.4 \%)$, France (58, $14.1 \%)$, UK $(57,13.9 \%)$, Italy $(56,13.6 \%)$, Spain $(56,13.6 \%)$, and Germany $(55,13.4 \%)$. The majority of physicians were psychiatrists (343/ $411,83.5 \%$ ), while the remainder were primary care physicians $(68 / 411,16.6 \%)$. Respondents had been qualified for a median of 15 years, dividing into experience groups of 3-10 years (101/411, 24.6\%), 11-20 years (209/411, 50.9\%) or $\geq 21$ years $(101 / 411,24.6 \%)$. The physicians reported that over $90 \%$ of their time, on average, was spent actively treating patients. In a typical month, they saw an average of 98 adults with MDD, around half of whom (47) were inadequately responding to ADT. On average, physicians estimated that $81.9 \%$ of their patients with MDD were receiving treatment for an acute episode, comprising $41.1 \%$ who were responding adequately, $19.8 \%$ with some unresolved symptoms but not requiring a change to their treatment regimen, and $21.0 \%$ with an inadequate response that did require a treatment change. The remaining patients (18.1\%) were receiving maintenance therapy to prevent further relapse.

\section{Treatments}

Physicians completed the survey for a total of 4018 patients whom they identified as having MDD and an inadequate response to current treatment. Prior to the current consultation, $1880 / 4018$ patients (46.8\%) were receiving monotherapy, mostly with an ADT (1766/ $4018,44.0 \%)$, and $2137 / 4018$ patients (53.2\%) were on combination therapy (see Table 1 ). The combinations most frequently mentioned were an ADT with a benzodiazepine (492/4018, $12.2 \%)$ or an ADT with an antipsychotic (294/ 4018, 7.3\%). Other combinations (860/4018, $21.4 \%$ ) comprised various combinations of three or more drugs. Antipsychotics were being prescribed to $40 / 4018$ patients $(1.0 \%)$ as monotherapy and 294/4018 (7.3\%) as an adjunctive to an ADT.

At the current consultation, physicians were most likely to switch the ADT to a different drug or add a new adjunctive drug (see Table 2). A treatment change involving an adjunctive antipsychotic was considered for 961/4018 patients (23.9\%). Around half of these patients 
Table 1 Current pharmacological treatment

\begin{tabular}{|c|c|c|c|c|}
\hline \multirow[t]{2}{*}{ Treatment, $n(\%)$} & \multicolumn{2}{|c|}{ Adjunctive antipsychotic patients ${ }^{\mathrm{a}}(N=961)$} & \multirow{2}{*}{$\begin{array}{l}\text { Non-adjunctive } \\
\text { antipsychotic } \\
\text { patients }(N=3057)\end{array}$} & \multirow{2}{*}{$\begin{array}{l}\text { All patients } \\
(N=4018)\end{array}$} \\
\hline & Considered $(n=447)$ & Prescribed $(n=514)$ & & \\
\hline Monotherapy & $129(28.9)$ & $172(33.5)$ & $1579(51.7)$ & $1880(46.8)$ \\
\hline SSRI & $81(18.1)$ & $98(19.1)$ & $1017(33.3)$ & $1196(29.8)$ \\
\hline SNRI & $23(5.1)$ & $44(8.6)$ & $292(9.6)$ & $359(8.9)$ \\
\hline Tricyclic & $6(1.3)$ & $7(1.4)$ & $46(1.5)$ & $59(1.5)$ \\
\hline Other ADT & $13(2.9)$ & $13(2.5)$ & $126(4.1)$ & $152(3.8)$ \\
\hline Hypnotic/anxiolytic & $0(0.0)$ & $1(0.2)$ & $26(0.9)$ & $27(0.7)$ \\
\hline Atypical AP & $3(0.7)$ & $5(1.0)$ & $32(1.0)$ & $40(1.0)$ \\
\hline Other $^{\mathrm{b}}$ & $3(0.7)$ & $4(0.8)$ & $40(1.3)$ & $47(1.2)$ \\
\hline Combination therapy & $318(71.1)$ & $342(66.5)$ & $1477(48.3)$ & $2137(53.2)$ \\
\hline 2 ADTs & $32(7.2)$ & $25(4.9)$ & $169(5.5)$ & $226(5.6)$ \\
\hline $\mathrm{ADT}+\mathrm{BZ}$ & $52(11.6)$ & $63(12.3)$ & $377(12.3)$ & $492(12.2)$ \\
\hline $\mathrm{ADT}+\mathrm{AP}$ & $45(10.1)$ & $60(11.7)$ & $189(6.2)$ & $294(7.3)$ \\
\hline $\mathrm{ADT}+\mathrm{MS}$ & $9(2.0)$ & $4(0.8)$ & $36(1.2)$ & $49(1.2)$ \\
\hline ADT + other $^{\mathrm{b}}$ & $19(4.3)$ & $24(4.7)$ & $173(5.7)$ & $216(5.4)$ \\
\hline Other combination $^{c}$ & $161(36.0)$ & $166(32.3)$ & $533(17.4)$ & $860(21.4)$ \\
\hline No drug treatment & $0(0.0)$ & $0(0.0)$ & $1(0.03)$ & $1(0.02)$ \\
\hline
\end{tabular}

$A D T$ Antidepressant treatment, $A P$ antipsychotic, $B Z$ benzodiazepine, $M S$ mood stabilizer, $S N R I$ serotonin noradrenaline reuptake inhibitor, $S S R I$ selective serotonin reuptake inhibitor

a Patients for whom the physician considered prescribing or prescribed an adjunctive antipsychotic, either as a new adjunctive treatment or as a switch from the patient's current adjunctive treatment

b Other drugs included lithium, valproate, St John's wort, and other pharmacological treatments

c Various combinations of three or more drugs

were actually prescribed an adjunctive antipsychotic $(514 / 4018,12.8 \%)$. This was prescribed as a new adjunctive drug for $343 / 4018$ patients $(8.5 \%)$ and as a switch of an existing adjunctive drug for $173 / 4018$ patients (4.3\%). Two patients were each prescribed two new adjunctive antipsychotics and one patient was prescribed a new adjunctive antipsychotic and was switched from a currently prescribed drug to an antipsychotic. In addition, antipsychotic monotherapy was newly prescribed for 55/4018 patients (1.4\%).
Physicians appeared to be more likely to consider prescribing or actually prescribe adjunctive antipsychotics for patients who were already on combination therapy than those on monotherapy (see Table 1).

When considering the influence of physician experience on this treatment decision, experienced physicians ( $>15$ years in practice, based on median experience) prescribed adjunctive antipsychotics in 242/1779 (13.6\%) patients compared with 272/2239 (12.2\%) patients prescribed adjunctive antipsychotics 
Table 2 Treatment changes at current consultation

\begin{tabular}{|c|c|c|c|c|}
\hline \multirow[t]{2}{*}{ Treatment change, $n(\%)^{\mathrm{b}}$} & \multicolumn{2}{|c|}{ Adjunctive antipsychotic patients ${ }^{\mathrm{a}}(N=961)$} & \multirow{2}{*}{$\begin{array}{l}\text { Non-adjunctive } \\
\text { antipsychotic } \\
\text { patients }(N=3057)\end{array}$} & \multirow{2}{*}{$\begin{array}{l}\text { All patients } \\
(N=4018)\end{array}$} \\
\hline & Considered $(n=447)$ & Prescribed $(n=514)$ & & \\
\hline Switch antidepressant drug & $243(54.4)$ & $94(18.3)$ & $1176(38.5)$ & $1513(37.7)$ \\
\hline New adjunctive drug ${ }^{c}$ & $113(25.3)$ & $366(71.2)$ & $763(25.0)$ & $1242(30.9)$ \\
\hline Switch adjunctive drug & $29(6.5)$ & $190(37.0)$ & $445(14.6)$ & $664(16.5)$ \\
\hline Other action, e.g., dose change & $106(23.7)$ & $8(1.6)$ & $972(31.8)$ & $1086(27.0)$ \\
\hline
\end{tabular}

a Patients for whom the physician considered prescribing or prescribed an adjunctive antipsychotic, either as a new adjunctive treatment or as a switch from the patient's current adjunctive treatment

b Patients may have had more than one type of treatment change

c Including antidepressant drugs, hypnotics, anxiolytics, atypical antipsychotics, lithium, valproate, St John's wort, and other drugs

by less experienced physicians. When this was re-examined for a cut-point of more or less than 10 years' experience, the difference remained marginal, with a reversed numerical difference (12.4\% versus $13.8 \%$, respectively), suggesting experience did not affect the decision to prescribe adjunctive antipsychotics. It was also unaffected by region; US physicians prescribed for $166 / 1198(13.9 \%)$ patients, compared with $348 / 2820 \quad(12.3 \%)$ patients prescribed adjunctive antipsychotics by European physicians.

\section{Patient Demographics and Disease History}

The study population was spread across all age categories from $18-24$ years to $\geq 65$ years (see Table 3). Slightly more than half of the patients were female. Compared with patients who were not considered for an adjunctive antipsychotic, there was some evidence that those who were considered for or prescribed this treatment had more previous MDEs, longer duration of the current MDE, greater CGI-S score both at ADT initiation and the current consultation, and more previous treatment changes (see Table 3).

\section{Employment Status and Patient Functioning}

Data regarding employment status and patient functioning were collected for patients who were considered for but not prescribed an adjunctive antipsychotic and those who were prescribed an adjunctive antipsychotic. Employment status was similar in both groups: among patients who were prescribed an adjunctive antipsychotic, 109/514 (21.2\%) were working full-time and 79/514 (15.4\%) were working part-time, compared with 91/446 (20.4\%) and 58/446 (13.0\%), respectively, in those considered for an adjunctive antipsychotic. However, absenteeism was significantly higher in those who were prescribed compared with those who were considered for an adjunctive antipsychotic (prescribed: $n=154$, mean 12.2 days/month versus considered: $n=128, \quad$ mean 9.3 days/month; $P<0.05)$. Additionally, the number of days/month during which patients did not perform their usual daily tasks at their usual level (presenteeism) was significantly higher in patients prescribed than those considered for an adjunctive antipsychotic 
Table 3 Demographic and disease characteristics

\begin{tabular}{|c|c|c|c|c|}
\hline & \multicolumn{2}{|c|}{$\begin{array}{l}\text { Adjunctive antipsychotic patients }{ }^{\mathrm{a}} \\
(N=961)\end{array}$} & \multirow{2}{*}{$\begin{array}{l}\text { Non-adjunctive } \\
\text { antipsychotic } \\
\text { patients } \\
(N=3057)\end{array}$} & \multirow[t]{2}{*}{$\begin{array}{l}\text { All patients } \\
(N=4018)\end{array}$} \\
\hline & $\begin{array}{l}\text { Considered } \\
(n=447)\end{array}$ & $\begin{array}{l}\text { Prescribed } \\
(n=514)\end{array}$ & & \\
\hline \multicolumn{5}{|l|}{ Age, $n(\%)$} \\
\hline $18-24$ years & $49(11.0)$ & $48(9.3)$ & $341(11.2)$ & $438(10.9)$ \\
\hline $25-34$ years & $73(16.3)$ & $94(18.3)$ & $637(20.8)$ & $804(20.0)$ \\
\hline $35-44$ years & $77(17.2)$ & $107(20.8)$ & $622(20.4)$ & $806(20.1)$ \\
\hline $45-54$ years & $115(25.7)^{b}$ & $123(23.9)$ & $633(20.7)$ & $871(21.7)$ \\
\hline 55-64 years & $73(16.3)$ & $81(15.8)$ & $494(16.2)$ & $648(16.1)$ \\
\hline$\geq 65$ years & $60(13.4)$ & $61(11.9)$ & $330(10.8)$ & $451(11.2)$ \\
\hline Mean, years & $45.6^{\mathrm{b}}$ & 44.7 & 43.8 & 44.1 \\
\hline \multicolumn{5}{|l|}{ Gender, $n(\%)$} \\
\hline Male & $187(41.8)$ & $241(46.9)^{b}$ & $1287(42.1)$ & $1715(42.7)$ \\
\hline Female & $260(58.2)$ & $273(53.1)$ & $1770(57.9)$ & $2303(57.3)$ \\
\hline Age of onset of first MDE, $n(\%)$ & $(n=405)$ & $(n=471)$ & $(n=2840)$ & $(n=3716)$ \\
\hline $18-24$ years & $114(28.2)$ & $111(23.6)$ & $715(25.2)$ & $940(25.3)$ \\
\hline $25-34$ years & $121(29.9)$ & $160(34.0)$ & $906(31.9)$ & $1187(31.9)$ \\
\hline $35-44$ years & $75(18.5)$ & $94(20.0)$ & $601(21.2)$ & $770(20.7)$ \\
\hline $45-54$ years & $54(13.3)$ & $60(12.7)$ & $324(11.4)$ & $438(11.8)$ \\
\hline $55-64$ years & $23(5.7)$ & $26(5.5)$ & $186(6.6)$ & $235(6.3)$ \\
\hline$\geq 65$ years & $18(4.4)$ & $20(4.3)$ & $108(3.8)$ & $146(3.9)$ \\
\hline Mean, years & 32.4 & 33.2 & 33.1 & 33.0 \\
\hline \multicolumn{5}{|c|}{ Number of MDEs since diagnosis, $n(\%)$} \\
\hline 1 & $67(15.0)$ & $89(17.3)$ & $824(27.0)$ & $980(24.4)$ \\
\hline 2 & $110(24.6)$ & $129(25.1)$ & $797(26.1)$ & $1036(25.8)$ \\
\hline 3 & $94(21.0)$ & $101(19.7)$ & $542(17.7)$ & 737 (18.3) \\
\hline 4 & $46(10.3)$ & $58(11.3)^{b}$ & $256(8.4)$ & $360(9.0)$ \\
\hline$\geq 5$ & $130(29.1)^{b}$ & $137(26.7)^{b}$ & $638(20.9)$ & $905(22.5)$ \\
\hline Mean & $4.3^{\mathrm{b}}$ & 3.7 & 3.6 & 3.7 \\
\hline \multicolumn{5}{|l|}{ Duration of current MDE, $n(\%)$} \\
\hline$<1$ month & $62(13.9)$ & $69(13.4)$ & $532(17.4)$ & $663(16.5)$ \\
\hline $1-3$ months & $159(35.6)$ & $208(40.5)$ & $1215(39.7)$ & $1582(39.4)$ \\
\hline $3-6$ months & $145(32.4)^{b}$ & $155(30.2)$ & $822(26.9)$ & $1122(27.9)$ \\
\hline
\end{tabular}


Table 3 continued

\begin{tabular}{|c|c|c|c|c|}
\hline & \multicolumn{2}{|c|}{$\begin{array}{l}\text { Adjunctive antipsychotic patients }{ }^{\mathrm{a}} \\
(N=961)\end{array}$} & \multirow{2}{*}{$\begin{array}{l}\text { Non-adjunctive } \\
\text { antipsychotic } \\
\text { patients } \\
(N=3057)\end{array}$} & \multirow[t]{2}{*}{$\begin{array}{l}\text { All patients } \\
(N=4018)\end{array}$} \\
\hline & $\begin{array}{l}\text { Considered } \\
(n=447)\end{array}$ & $\begin{array}{l}\text { Prescribed } \\
(n=514)\end{array}$ & & \\
\hline 6-9 months & $28(6.3)$ & $40(7.8)$ & $168(5.5)$ & $236(5.9)$ \\
\hline $9-12$ months & $27(6.0)$ & $25(4.9)$ & $168(5.5)$ & $220(5.5)$ \\
\hline$\geq 12$ months & $26(5.8)$ & $17(3.3)$ & $152(5.0)$ & $195(4.9)$ \\
\hline Mean, months & $5.5^{\mathrm{b}}$ & 4.6 & 4.9 & 5.0 \\
\hline \multicolumn{5}{|l|}{ CGI-S score, $n(\%)$} \\
\hline \multicolumn{5}{|l|}{ At $\mathrm{ADT}$ initiation } \\
\hline Mild (1-2) & $7(1.6)$ & $7(1.4)$ & $55(1.8)$ & $69(1.7)$ \\
\hline Moderate (3-5) & $270(60.4)$ & $294(57.2)$ & $2198(71.9)$ & $2762(68.7)$ \\
\hline Severe $(6-7)$ & $166(37.1)^{\mathrm{b}}$ & $208(40.5)^{b}$ & $759(24.8)$ & $1133(28.2)$ \\
\hline Unknown & $4(0.9)$ & $5(1.0)$ & $45(1.5)$ & $54(1.3)$ \\
\hline \multicolumn{5}{|l|}{ Today } \\
\hline Mild (1-2) & $66(14.8)$ & $60(11.7)$ & $691(22.6)$ & $817(20.3)$ \\
\hline Moderate (3-5) & $338(75.6)$ & $380(73.9)$ & $2196(71.8)$ & $2914(72.5)$ \\
\hline Severe $(6-7)$ & $43(9.6)^{b}$ & $74(14.4)^{\mathrm{b}}$ & $170(5.6)$ & $287(7.1)$ \\
\hline \multicolumn{5}{|l|}{ Comorbidities, $n$ (\%) } \\
\hline None & $55(12.3)$ & $74(14.4)$ & $572(18.7)$ & $701(17.5)$ \\
\hline Any psychiatric comorbidity ${ }^{c}$ & $220(49.2)$ & $276(53.7)$ & $1279(41.8)$ & $1775(44.2)$ \\
\hline Any somatic comorbidity ${ }^{\mathrm{c}}$ & $306(68.5)$ & $339(66.0)$ & $1819(59.5)$ & $2464(61.3)$ \\
\hline No current non-pharmacological treatment, $n$ (\%) & $159(35.6)$ & $176(34.2)$ & $1270(41.5)$ & $1605(40.0)$ \\
\hline Mean number of previous treatment changes & $5.3^{\mathrm{b}}$ & $4.9^{\mathrm{b}}$ & 3.7 & 4.0 \\
\hline
\end{tabular}

$A D T$ Antidepressant treatment, CGI-S clinical global impression-severity, $M D E$ major depressive episode

a Patients for whom the physician considered prescribing or prescribed an adjunctive antipsychotic

b $P<0.05$ versus non-adjunctive antipsychotic group (patients who were not considered for or prescribed an adjunctive antipsychotic)

c Psychiatric comorbidities included generalized anxiety disorder, panic disorder, social anxiety disorder, post-traumatic stress disorder, attention deficit hyperactivity disorder, bipolar disorder, schizophrenia, alcohol or drug use disorder and personality disorder; somatic comorbidities included chronic fatigue syndrome, fibromyalgia, neuropathic pain, irritable bowel syndrome, hypertension, hypercholesterolemia, insomnia, obesity, migraine, diabetes, osteoarthritis, asthma, chronic obstructive pulmonary disease, chronic pain, aches and pains, thyroid disorder and other

(prescribed: $n=376$, mean 17.4 days/month versus considered: $n=317$, mean 13.9 days/ month; $P<0.05)$.
Physicians considered MDD to have a great impact on family relationships in a significantly larger proportion of patients prescribed $(n=514)$ 
than considered for $(n=447)$ an adjunctive antipsychotic $(54.3 \%$ versus $43.2 \%, P<0.05)$. In both groups, MDD was considered to have a great impact, for the majority of patients, on social relationships (59.9\% versus $55.7 \%)$ and involvement in hobbies, interests or voluntary work (56.0\% versus $55.3 \%)$.

\section{Factors Influencing Prescription} of an Adjunctive Antipsychotic

The most common reason given by physicians for the actual prescription of an adjunctive antipsychotic was better efficacy or symptom control (52\%, Fig. 1). Specific drug features that were mentioned included non-sedative calming (20\%), sedation (16\%), and activation (14\%). When physicians were asked to identify their top three influences on the treatment decision, the highest ranked factors overall were their familiarity with or trust in the drug, the patient's level of functioning, a better tolerability profile, and the patient's history of MDD.

The most common reason that physicians gave for not prescribing an adjunctive antipsychotic or for not prescribing this treatment earlier was a preference to wait to see if the symptoms improved (1010/3538,

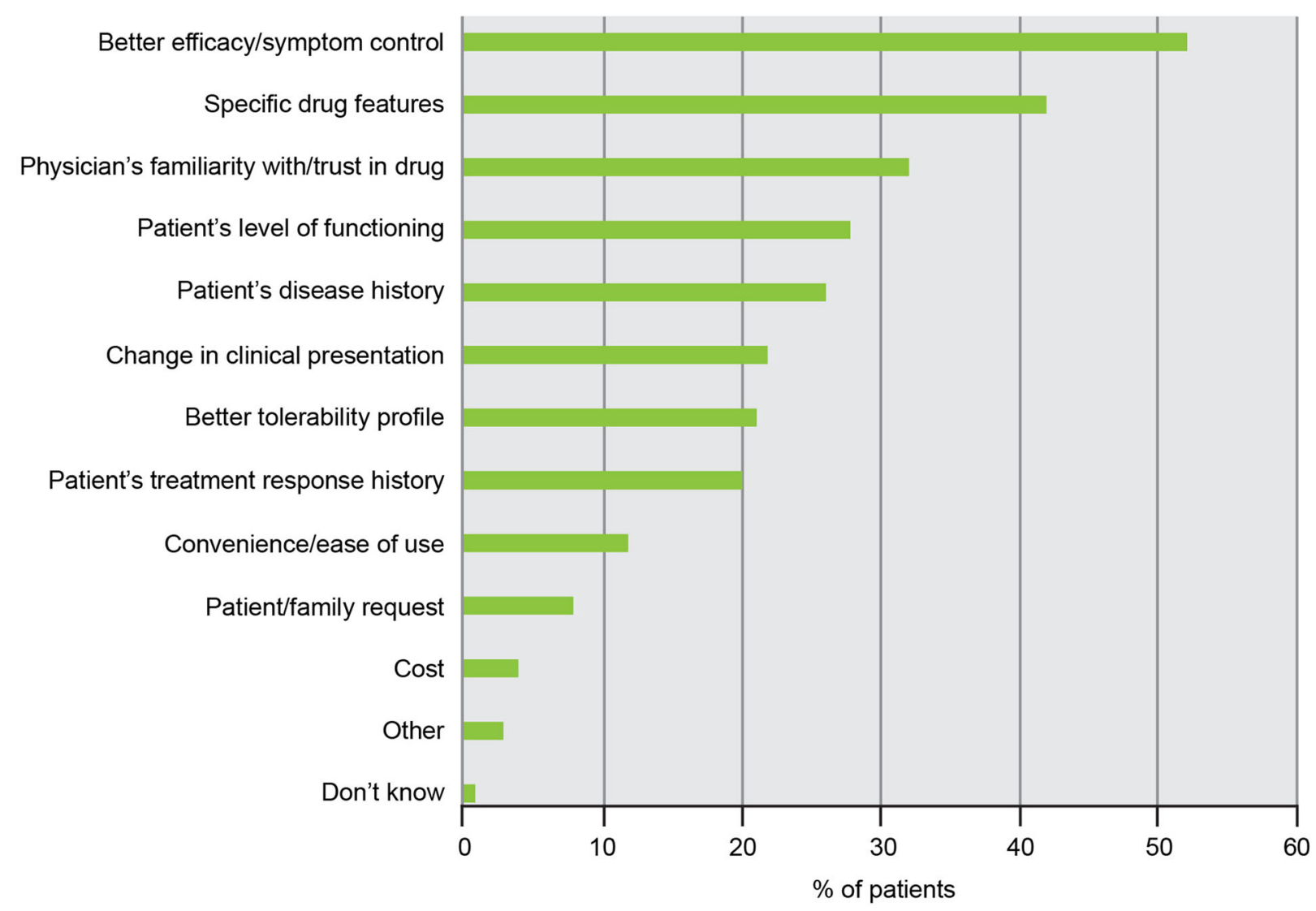

Fig. 1 Physician's reasons for deciding to prescribe an adjunctive antipsychotic. Drugs prescribed: aripiprazole $(n=174)$, quetiapine $\mathrm{XR} \quad(n=103)$, quetiapine IR $(n=96)$, olanzapine $(n=57)$, risperidone $(n=52)$, other antipsychotic $(n=22)$, lurasidone $(n=12)$, iloperidone $(n=1)$. Number of patients $=514$; total number of drugs prescribed $=517$, two patients were each prescribed two new adjunctive antipsychotics and one patient was prescribed a new adjunctive antipsychotic and was switched from a currently prescribed drug to an antipsychotic 


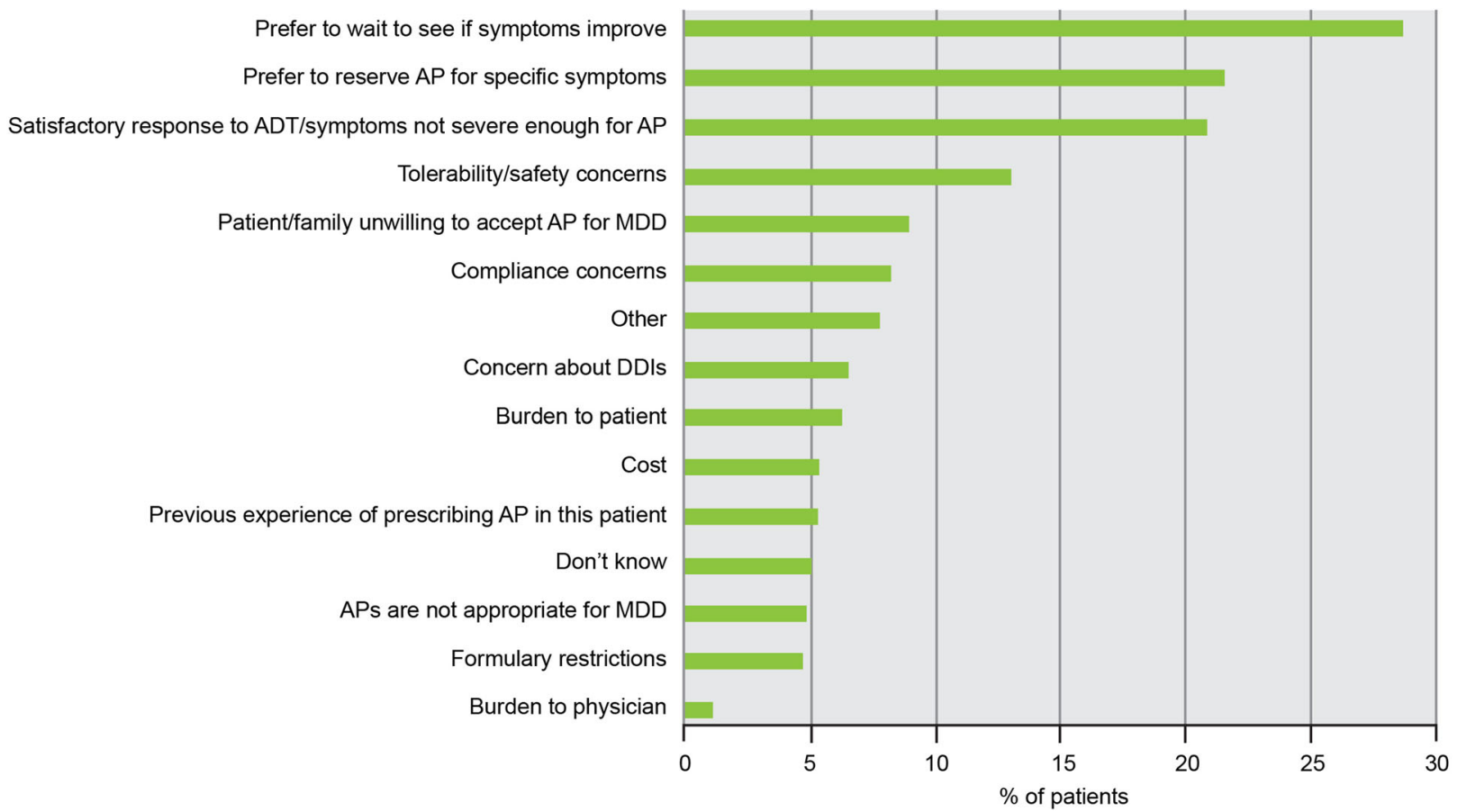

Fig. 2 Physician's reasons for not prescribing an adjunctive antipsychotic in patients who were not considered for this treatment and those who were considered but not prescribed this treatment, and for not prescribing earlier in those who were prescribed this treatment. Number of responses $=3538$, comprising data for 2577 patients who

$28.6 \%$, based on the number of responses; Fig. 2); this appeared to be driven by less experienced physicians (611/1966, 31.1\%) more than experienced physicians (399/1572, $25.4 \%)$. Tolerability or safety concerns had prevented or delayed prescription of an adjunctive antipsychotic for $458 / 3538$ patients (12.9\%) and again this was a greater concern for less experienced physicians (280/1966, 14.2\% versus $178 / 1572,11.3 \%)$. Tolerability was also more of a concern for US than European physicians (167/1018, 16.4\% versus 291/2520, $11.6 \%)$. The physicians' main concerns were weight gain $(275 / 458,60.0 \%)$, other metabolic side effects (264/458, 57.6\%), extrapyramidal symptoms (EPS; 198/458, 43.2\%), sedation (146/ 458, 31.9\%), akathisia (117/458, 25.6\%), and prolactin-related side effects (100/458, 21.8\%). were not considered for an adjunctive antipsychotic, 447 patients who were considered for but not prescribed this treatment, and 514 patients who were prescribed this treatment (i.e., reasons for not prescribing earlier). $A D T$ antidepressant treatment, $A P$ antipsychotic, $D D I$ drugdrug interaction, $M D D$ major depressive disorder

\section{Symptoms Influencing Antipsychotic Prescribing}

Overall, 295/411 physicians (71.8\%) indicated for at least one of their patients that they preferred to reserve antipsychotics for patients with MDD who had specific symptoms. When this group of physicians was asked which symptoms they preferred to reserve antipsychotics for, those symptoms most frequently identified were: psychotic symptoms $(196 / 295,66.4 \%)$, psychomotor agitation (104/295, 35.3\%), hostility (97/295, $32.9 \%)$, irritability $(85 / 295,28.8 \%)$, impulsivity $(83 / 295,28.1 \%)$, and anger bursts $(80 / 295$, $27.1 \%$; Fig. 3). These symptoms were relatively consistently selected across regions: US physicians identified their top symptoms as 


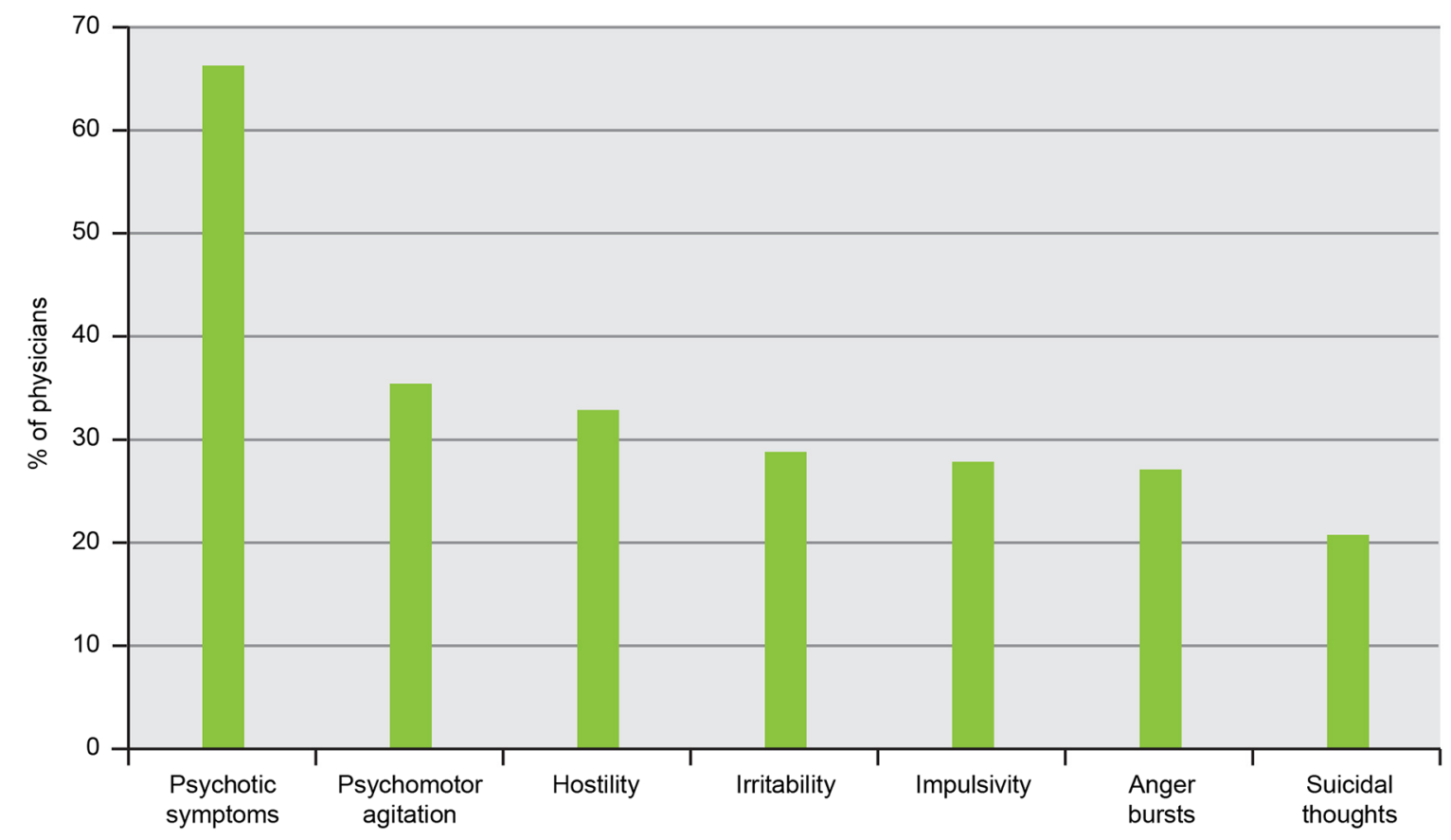

Fig. 3 Physician's indication of the symptoms that they preferred to reserve antipsychotics for in patients with MDD. Symptoms shown are those selected by $\geq 20 \%$ of physicians. Number of responses $=295$, comprising

psychotic symptoms (41/70, 58.6\%), hostility (27/70, 38.6\%), psychomotor agitation (23/70, $32.9 \%)$, irritability $(20 / 70,28.6 \%)$, anger bursts $(19 / 70,27.1 \%)$ and impulsivity $(15 / 70,21.4 \%)$, with the same overall trends seen across the European region as well.

Anxious mood and irritability were mentioned more often as trigger symptoms by physicians who actually prescribed adjunctive antipsychotics to their survey patients than those who did not (anxious mood: 14/60, $23.3 \%$ versus $15 / 235,6.4 \%$; irritability: $26 / 60$, $43.3 \%$ versus $59 / 235,25.1 \%$ ). The most frequently reported symptoms that actually led to prescription or consideration of an adjunctive antipsychotic for patients in the survey were depressed mood (259/961, 27.0\%) and anxious $\operatorname{mood}(194 / 961,20.2 \%$; Fig. 4), which were mostly rated as moderate or severe. Among physicians who indicated for at least one of their patients that they preferred to reserve antipsychotics for patients with MDD who had specific symptoms. $M D D$ major depressive disorder

patients who were actually prescribed an adjunctive antipsychotic, 87/514 (16.9\%) had two symptoms, and 200/514 (38.9\%) had three or more symptoms, that led to this prescription.

Differences were seen in the symptom profile captured by coded and open formats; most symptoms were mentioned less often in the open format. In the coded format (symptom checklist), the most frequently selected symptoms were: depressed mood (47\%), anhedonia (31\%), and anxious mood (31\%). In the open format (text box), the most frequently mentioned symptoms were: anxious mood (17\%), psychotic symptoms (15\%), and depressed mood (14\%). Of the 145 patients who were prescribed an adjunctive antipsychotic due to or partly due to anxious mood, 24 (16.6\%) also had a benzodiazepine added to their treatment regimen. 


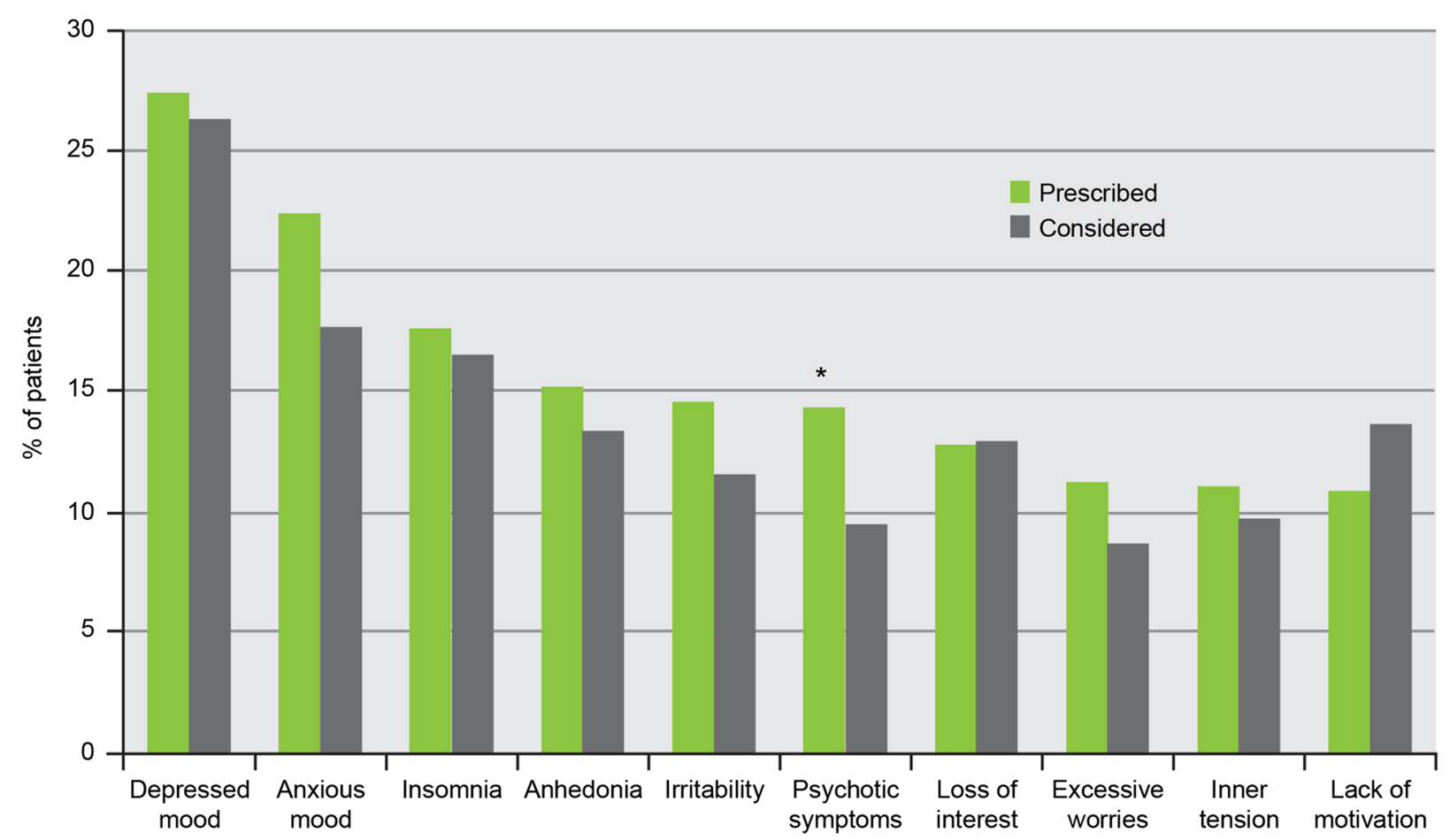

Fig. 4 Physician's indication of symptoms that actually led to consideration for, or prescription of, an adjunctive antipsychotic in $\geq 10 \%$ of patients. ${ }^{*} P<0.05$ for considered

\section{DISCUSSION}

This case review study was conducted to determine which patients with MDD and an inadequate response to ADT are prescribed an adjunctive antipsychotic and the key factors that influence this decision. The study was conducted in a real-world setting to provide qualitative information on treatment decision making in clinical practice across Europe and the USA. The psychiatrists and primary care physicians who participated in the study had extensive experience in active clinical management of patients with MDD. In accordance with previous reports suggesting that only $50-60 \%$ of patients with MDD respond to first-line treatment [1], the physicians estimated that around half of their caseload of patients with MDD were not responding adequately to their current versus prescribed. Number of responses $=961$, comprising data for 447 patients who were considered for and 514 patients who were prescribed an adjunctive antipsychotic

treatment regimen. Neither MDD diagnosis nor inadequate responses were defined for the physicians, to make this study as naturalistic as possible, but the data gathered here provide some interesting insights into what aspects of patient presentation are associated with physician perceptions of MDD and inadequate response. Interestingly, among the patients with MDD and an inadequate response to ADT who were profiled for this study, around half were already receiving combination therapy.

Augmentation or combination strategies are recommended by treatment guidelines for patients with MDD who experience partial or no response to ADT [11]. Among strategies to augment response with non-ADTs, adjunctive second-generation antipsychotics are supported by the strongest evidence [1]. In this study, a treatment change involving an adjunctive antipsychotic was considered for $23.9 \%$ of the 
patients and actually made for $12.8 \%$. Patients who were prescribed adjunctive antipsychotics had more severe disease than those who did not receive this treatment, as evidenced by their disease and treatment history, and CGI-S scores both at initiation of treatment and the current consultation. Consistent with this finding, a retrospective study using data from patient records reported that patients with severe depression had an increased likelihood of augmentation with second-generation antipsychotics [10]. In our study, functional impairment was also associated with adjunctive antipsychotic prescribing. Although employment status was similar in patients who were considered for and prescribed adjunctive antipsychotics, it is interesting that the patients who were actually prescribed this treatment tended to take more days off work and to experience more time during which they were unable to perform their daily tasks at the usual level. It is noteworthy that physicians considered MDD to have a great impact on family relationships, social relationships and involvement in hobbies, interests and voluntary work, in more than half of the patients who were prescribed adjunctive antipsychotics, providing further evidence that functional impairment guides the treatment decision process.

The most common reason given by the physicians for adjunctive antipsychotic prescribing was to achieve better efficacy or control of symptoms. Physicians who stated that they reserved antipsychotics for patients with specific symptoms indicated that key triggers would be psychotic symptoms, and arousal symptoms such as psychomotor agitation, hostility, irritability, impulsivity, and anger bursts. That those physicians who reserved antipsychotic treatment were triggered to add an antipsychotic by presence of psychotic symptoms is perhaps not surprising, but does raise the possibility that this is uncovering the well-documented underdiagnosis of bipolar spectrum disorder by these physicians [12]. Anxious mood and irritability were more likely to be mentioned as trigger symptoms by physicians who actually prescribed adjunctive antipsychotics to survey patients than those who did not prescribe. For the patients included in this survey, unsurprisingly, moderate-to-severe depression was a key influence on the treatment decision; interestingly, moderate-to-severe anxiety was also an important factor that led the physician to consider adjunctive antipsychotics. It is recognized that anxiety symptoms are common in patients with MDD [13, 14] and are associated with greater illness severity, unfavorable course of illness, impaired functioning, and poorer health-related quality of life [15-17]. Other symptoms which led to the prescription of an adjunctive antipsychotic included core features of MDD such as insomnia and anhedonia as well as irritability and psychotic symptoms. More symptoms were reported when the physician was provided with a symptom checklist rather than an open text box, which perhaps suggests that physicians are considering a broad array of issues with each patient, while focusing on key symptoms for treatment decisions.

Current evidence supports the use of adjunctive antipsychotics in patients with MDD and an inadequate response to ADT [1, $8,18]$. Randomized, placebo-controlled clinical studies have demonstrated significant reductions in clinician-rated symptoms of depression in patients with MDD who were treated with adjunctive antipsychotics. Augmentation with antipsychotics may be effective in patients with an incomplete response to ADT as well as in those who are 
treatment resistant. The biological rationale for using adjunctive antipsychotics stems from the broad receptor-binding profiles of these medications at dopaminergic, noradrenergic, and serotonergic receptors $[1,8]$. For instance, increased dopamine transmission in the mesocortical dopamine pathway [8] may explain the potential of these therapies to be more effective than adding another ADT or switching to a treatment with less profoundly different binding profiles.

Obstacles to adjunctive antipsychotic prescribing were also examined in this study. Physicians indicated that they often preferred to wait for a while to see if the symptoms improved before prescribing an adjunctive antipsychotic. Concern around the side effects of antipsychotics was identified, particularly regarding weight gain, related metabolic effects, sedation, akathisia and EPS. It is understandable that physicians may be cautious in prescribing antipsychotics since it is well known that certain drugs in this class are associated with specific side effects, probably linked to their pharmacological profile. For example, quetiapine is associated with sedation [19] and aripiprazole with akathisia [20]. Selection of an antipsychotic for an individual patient should be guided by the patient's clinical characteristics and symptom profile.

This study has a number of limitations. It should be noted that the data were not collected as part of a prospective, controlled trial and the survey responses were based on the physician's recent memory of the consultation and the patient's case notes. Many of the data collected were estimated or categorical. Nevertheless, this study provides real-world evidence of physicians' perceptions and clinical practice patterns that cannot be determined in a randomized, controlled trial.

\section{CONCLUSIONS}

This real-world case review study suggests that the decision to prescribe an adjunctive antipsychotic for a patient with MDD and an inadequate response to ADT is influenced by a broad spectrum of factors. Unsurprisingly, physicians tended to reserve adjunctive antipsychotics for patients with more severe illness and greater functional impairment, and those who had already failed a number of treatment options. Consideration was also given to the patient's symptom profile. As well as depressive features, symptoms such as anxiety, psychotic symptoms, and irritability were likely to prompt physicians to prescribe adjunctive antipsychotics.

\section{ACKNOWLEDGMENTS}

This study was conducted by Ashfield Insight and Performance (Macclesfield, UK). Sponsorship for this study, article processing charges, and the open access fee were funded by Otsuka Pharmaceutical Development \& Commercialization, Inc. (Princeton, USA) and H. Lundbeck A/S (Valby, Denmark). All named authors meet the International Committee of Medical Journal Editors (ICMJE) criteria for this manuscript, take responsibility for the integrity of the work as a whole, and have given final approval to the version to be published. Writing support in the preparation of this manuscript was provided by Jennifer Stewart of QXV Communications, an Ashfield business, part of UDG Healthcare plc, Macclesfield, UK. Support for this assistance was funded by Otsuka Pharmaceutical Development \& Commercialization, Inc. (Princeton, USA) and H. Lundbeck A/S (Valby, Denmark). 
Conflict of interest. R. S. McIntyre has received research grants from Eli Lilly, JanssenOrtho, Shire, AstraZeneca, Pfizer, Lundbeck, Forest, Sunovion, Bristol-Myers Squibb, Stanley Medical Research Institute, National Alliance for Research on Schizophrenia and Depression (NARSAD), National Institutes of Mental Health, and Canadian Institutes of Health Research; has served on advisory boards for Bristol-Myers Squibb, Eli Lilly, Lundbeck, Pfizer, Shire, Merck, Sunovion, Otsuka, Forest, and Takeda; and has received speakers' fees from Janssen-Ortho, AstraZeneca, Eli Lilly, Lundbeck, Merck, Pfizer, Otsuka, Sunovion, Forest, and Takeda. E. Weiller is an employee of $\mathrm{H}$. Lundbeck A/S.

Compliance with ethics guidelines. The analysis in this article is based on patient record forms, and does not involve any new studies with human or animal subjects performed by any of the authors. Consequently, institutional review board approval and patient informed consent were not required.

Open Access. This article is distributed under the terms of the Creative Commons Attribution Noncommercial License which permits any noncommercial use, distribution, and reproduction in any medium, provided the original author(s) and the source are credited.

\section{REFERENCES}

1. Connolly KR, Thase ME. If at first you don't succeed: a review of the evidence for antidepressant augmentation, combination and switching strategies. Drugs. 2011;71(1):43-64.

2. Moller HJ. Outcomes in major depressive disorder: the evolving concept of remission and its implications for treatment. World $\mathrm{J}$ Biol Psychiatry. 2008;9(2):102-14.
3. Thase ME. Treatment-resistant depression: prevalence, risk factors, and treatment strategies. J Clin Psychiatry. 2011;72(5):e18.

4. Fleurence R, Williamson R, Jing $\mathrm{Y}$, et al. A systematic review of augmentation strategies for patients with major depressive disorder. Psychopharmacol Bull. 2009;42(3):57-90.

5. Patkar AA, Pae CU. Atypical antipsychotic augmentation strategies in the context of guidelinebased care for the treatment of major depressive disorder. CNS Drugs. 2013;27(Suppl 1):S29-37.

6. Taneja C, Papakostas GI, Jing Y, Baker RA, Forbes RA, Oster G. Cost-effectiveness of adjunctive therapy with atypical antipsychotics for acute treatment of major depressive disorder. Ann Pharmacother. 2012;46(5):642-9.

7. Cha DS, McIntyre RS. Treatment-emergent adverse events associated with atypical antipsychotics. Expert Opin Pharmacother. 2012;13(11):1587-98.

8. Wright BM, Eiland EH III, Lorenz R. Augmentation with atypical antipsychotics for depression: a review of evidence-based support from the medical literature. Pharmacotherapy. 2013;33(3):344-59.

9. Turner P, Kantaria R, Young AH. A systematic review and meta-analysis of the evidence base for add-on treatment for patients with major depressive disorder who have not responded to antidepressant treatment: a European perspective. J Psychopharmacol. 2014;28(2):85-98.

10. Gersing KR, Sheehan JJ, Burchett B, et al. Use of augmentation agents for treating depression: analysis of a psychiatric electronic medical record data set. Psychiatr Serv. 2014;65(8):1062-5.

11. Nutt DJ, Davidson JR, Gelenberg AJ, et al. International consensus statement on major depressive disorder. J Clin Psychiatry. 2010;71 Suppl E1:e08.

12. Ghaemi SN, Ko JY, Goodwin FK. "Cade's disease" and beyond: misdiagnosis, antidepressant use, and a proposed definition for bipolar spectrum disorder. Can J Psychiatry. 2002;47(2):125-34.

13. Fava M, Rankin MA, Wright EC, et al. Anxiety disorders in major depression. Compr Psychiatry. 2000;41(2):97-102.

14. Zimmerman M, McDermut W, Mattia JI. Frequency of anxiety disorders in psychiatric outpatients with major depressive disorder. Am J Psychiatry. 2000;157(8):1337-40.

15. Fichter MM, Quadflieg N, Fischer UC, Kohlboeck G. Twenty-five-year course and outcome in anxiety 
and depression in the Upper Bavarian Longitudinal Community Study. Acta Psychiatr Scand. 2010;122(1):75-85.

16. Rhebergen D, Batelaan NM, de Graaf R, et al. The 7-year course of depression and anxiety in the general population. Acta Psychiatr Scand. 2011;123(4):297-306.

17. Zimmerman M, Chelminski I, Young D, Dalrymple $\mathrm{K}$, Walsh E, Rosenstein L. A clinically useful selfreport measure of the DSM-5 anxious distress specifier for major depressive disorder. J Clin Psychiatry. 2014;75(6):601-7.

18. Spielmans GI, Berman MI, Linardatos E, Rosenlicht NZ, Perry A, Tsai AC. Adjunctive atypical antipsychotic treatment for major depressive disorder: a meta-analysis of depression, quality of life, and safety outcomes. PLoS Med. 2013;10(3):e1001403.

19. Pae CU, Sohi MS, Seo HJ, et al. Quetiapine XR: current status for the treatment of major depressive disorder. Prog Neuropsychopharmacol Biol Psychiatry. 2010;34(7):1165-73.

20. Pae CU, Forbes A, Patkar AA. Aripiprazole as adjunctive therapy for patients with major depressive disorder: overview and implications of clinical trial data. CNS Drugs. 2011;25(2):109-27. 